\title{
Bifidobacterium infantis suppresses proinflammatory interleukin-17 production in murine splenocytes and dextran sodium sulfate-induced intestinal inflammation
}

\author{
SOICHI TANABE ${ }^{1}$, YUKI KINUTA ${ }^{1}$ and YASUO SAITO $^{2}$ \\ ${ }^{1}$ Graduate School of Biosphere Science, Hiroshima University, Kagamiyama, Higashi-Hiroshima, Hiroshima 739-8528; \\ ${ }^{2}$ Institute for Technical Research, Glico Dairy Products Co. Ltd., Musashino, Akishima, Tokyo 196-0021, Japan
}

Received February 22, 2008; Accepted April 2, 2008

DOI: 10.3892/ijmm_00000006

\begin{abstract}
Interleukin (IL)-17 acts as a potent inflammatory cytokine, and IL-17-producing cells (Th17 cells) have received much attention. However, the involvement of commensal and/or probiotic bacteria in IL-17 production has not been evaluated. In this study, we examined the suppressive effects of five bacteria species on IL-17 production in vitro and ex vivo. Among the five species studied, Bifidobacterium infantis inhibited IL-17 production but enhanced IL-27 production most potently in TGF- $\beta$ plus IL-6-stimulated murine splenocytes. B. infantis also inhibited IL-17 and eotaxin production from a dextran sodium sulfate-treated colon organ culture. The induction of IL-10 by $B$. infantis was observed both in the splenocytes and in the colon culture and was assumed, to a certain extent, to be important for suppressing IL-17 production. These findings suggest a novel immunomodulatory function of commensal bifidobacteria and further imply that these bacteria may be useful in the treatment of Th17-mediated diseases.
\end{abstract}

\section{Introduction}

Inflammatory bowel diseases (IBDs) such as Crohn's disease and ulcerative colitis are characterized by recurrent inflammation in the gastrointestinal tract (1). The histology associated with IBD includes inflammation of the intestinal mucosa with neutrophilic and other inflammatory cell infiltration (2). Elevated levels of proinflammatory cytokines such as tumor necrosis factor- $\alpha$ (TNF- $\alpha$ ) and interferon- $\gamma$ $(\mathrm{IFN}-\gamma)$ are critically involved in the pathogenesis of IBD. This has been demonstrated in experimental colitis models as well as in biopsies from patients with IBD (3).

Correspondence to: Dr Soichi Tanabe, Graduate School of Biosphere Science, Hiroshima University, Kagamiyama, HigashiHiroshima, Hiroshima 739-8528, Japan

E-mail: stanabe@hiroshima-u.ac.jp

Key words: interleukin-17, interleukin-27, Th17, Treg, bifidobacteria, intestinal inflammation
In addition, interleukin (IL)-17 has recently been found to be elevated in the intestinal tissue and serum of patients with IBD (4). IL-17 acts as a potent inflammatory cytokine in vitro and in vivo (5) and is produced by the newly identified $\mathrm{T}$ cell subset Th17 $(6,7)$ and other non-T cells. Transcription factor $\mathrm{ROR} \gamma \mathrm{t}$ has been identified as being involved in the generation of Th17 cells (8).

IL-17 has pleiotropic activities, one of which is to coordinate tissue inflammation by inducing the expression of proinflammatory cytokines such as IL-6 and TNF- $\alpha$; chemokines such as KC, MCP-1 and MIP-2; and matrix metalloproteases which mediate tissue infiltration and tissue destruction (5). IL-17 is also involved in the proliferation, maturation and chemotaxis of neutrophils (9). It has been shown that cytokines such as IL-2, IL-25 and IL-27 suppress Th17 function (10-12). In particular, IL-27, which effectively blocks IL-17 production and induces immunoregulatory IL-10 activity, has recently attracted much attention (13-16).

There is growing evidence to suggest that probiotic bacteria may have, in a species- or even strain-dependent manner, potential use as anti-inflammatory agents in certain chronic inflammatory diseases such as IBD $(17,18)$. On the basis of experimental data, the anti-inflammatory effects of probiotics may be, in part, a consequence of the modulation of the cytokine balance: down-regulation of proinflammatory (e.g., IL-12 and TNF- $\alpha$ ) cytokine production and/or stimulation of anti-inflammatory (e.g., IL-10) cytokine production (19). In addition, in Caco-2 cells, the deleterious effects of TNF- $\alpha$ on epithelial function can be prevented by certain lactobacilli (20-22). As commensal and probiotic bacteria are recognized by transmembrane pattern recognition receptors known as Toll-like receptors (TLRs) (23), the involvement of TLRs in the anti-inflammatory effects of these bacteria has been extensively studied. For example, Cario et al (24) reported that TLR2 stimulation could preserve tight junction-associated barrier assembly against intestinal inflammation through PI3K/Akt-mediated cell survival via MyD88. It has also been reported that apical TLR9 signaling in intestinal epithelial cells regulates inflammation and maintains colonic homeostasis (25). However, the effect of commensal and probiotic bacteria such as bifidobacteria on IL-17-producing cells has not yet been examined. 
The aim of the present study was to assess whether commensal bifidobacteria and probiotic lactobacilli suppressed IL-17 production in murine splenocytes and in dextran sodium sulfate (DSS)-induced intestinal inflammation. Here, we clearly show, for the first time, that Bifidobacterium infantis $\left(\mathrm{JCM} 1222^{\mathrm{T}}\right)$, one of the major commensal bacteria in the intestine of infants, suppressed IL-17 production both in splenocytes and colon organ culture.

\section{Materials and methods}

Reagents. RPMI-1640 medium, penicillin and streptomycin were purchased from Invitrogen (Carlsbad, CA, USA). Fetal bovine serum (FBS) was obtained from ICN Biomedicals (Osaka, Japan). DSS was purchased from Wako Pure Chemical Industries (Osaka, Japan). Both recombinant human TGF- $\beta$ and recombinant mouse IL- 6 were obtained from R\&D Systems (Minneapolis, MN, USA). GAM broth was purchased from Nissui Pharmaceutical (Tokyo, Japan), and MRS broth was purchased from Merck (Darmstadt, Germany). All other chemicals were of reagent grade.

Bacterial cultures. Three species of bifidobacteria (B. bifidum, $B$. catenulatum and B. infantis) and two species of lactobacilli (L. acidophilus and L. bulgaricus) were obtained from Riken Bioresource Center (Japan Collection of Microorganisms, Saitama, Japan). Bifidobacteria were cultured in GAM broth and lactobacilli were cultured in MRS broth, and cultures were incubated at $37^{\circ} \mathrm{C}$ for $18 \mathrm{~h}$ in an anaerobic bag (AnaeroPack, Mitsubishi Gas Chemical, Tokyo, Japan). The cell suspensions were washed with distilled water, incubated at $100^{\circ} \mathrm{C}$ for $50 \mathrm{~min}$, and then lyophilized. Subsequently, the heat-killed bacteria were added to the splenocyte culture or the colon organ culture as described below.

Splenocyte culture. Six-week-old female Balb/c mice were obtained from Charles River (Kanagawa, Japan) and sacrificed by cervical dislocation. The spleens were removed from three mice for each experiment, and the pooled splenocytes $\left(10^{7}\right.$ cells) were incubated with TGF- $3(2 \mathrm{ng} / \mathrm{ml})$ plus IL-6 (20 ng/ $\mathrm{ml})$ at $37^{\circ} \mathrm{C}$ for $72 \mathrm{~h}$ in $1 \mathrm{ml}$ of RPMI-1640 medium supplemented with $10 \%$ FBS, $10 \mu \mathrm{M}$ 2-mercaptoethanol, $10 \mathrm{mM}$ HEPES, penicillin and streptomycin. The heat-killed bacteria $\left(10^{7}\right.$ cells) were added to the culture. A culture without the addition of TGF- $\beta$ plus IL- 6 or heat-killed bacteria was included as a control. Culture supernatants were harvested and assayed for cytokines as described below.

Colon organ culture. Segments of the distal colon $(5 \mathrm{~cm}$ in length) were removed from at least three Balb/c mice (sixweek-old females), cut open longitudinally, and washed in phosphate-buffered saline. The colon was then further cut into strips $<0.3 \mathrm{~cm}^{2}$, and these were placed in a 24-well flatbottom culture plate containing the same RPMI-1640 medium as indicated above. Four strips each were incubated with DSS $(0.5,1$ and $2 \%)$ or TGF- $3(2 \mathrm{ng} / \mathrm{ml})$ plus IL-6 $(20 \mathrm{ng} / \mathrm{ml})$ at $37^{\circ} \mathrm{C}$ for $24 \mathrm{~h}$ in 0.5 or $1 \mathrm{ml}$ of the medium. The heat-killed bacteria ( $10^{5}$ cells) were added to the culture. A culture without the addition of DSS, TGF- $\beta$ plus IL- 6 or heat-killed bacteria was included as a control. Culture super- natants were harvested and assayed for cytokines and eotaxin as described below.

Cytokine assays. IL-17, IL-27 and eotaxin concentrations of the culture supernatants were measured by sandwich enzymelinked immunosorbent assay (ELISA) (R\&D Systems) according to the manufacturer's instructions (Figs. 1 and 2). For the evaluation of the effect of $B$. infantis on the cytokine profile of the culture supernatant, cytokine [IL-2, IL-4, IL-5, IL-10 and IL-12 (p70)] concentrations were determined by the microbead method using the Bio-Plex Suspension Array System (BioRad Laboratories, Hercules, CA, USA) (Tables I and II). Briefly, culture supernatants were incubated with beads conjugated with anti-IL-2, -IL-4, -IL-5, -IL-10 and -IL-12 (p70) antibody followed by a sandwich immunoassay using biotinylated secondary antibodies. The beads were washed three times during each incubation. Phycoerythrin-streptavidin was used as a reporter. The relative fluorescence units were detected by counting 100 beads with the Bio-Plex apparatus. Data were evaluated with the Bio-Plex Manager Software 3.0 (BioRad Laboratories) using 5PL curve fitting.

Statistical analysis. Statistical analysis was performed using one-way ANOVA followed by Tukey's post-hoc test.

\section{Results}

Effects of heat-killed bifidobacteria on IL-17 and IL-27 production in murine splenocytes. Prior to the analyses of the regulatory effects of bifidobacteria and lactobacilli on IL-17 production in the inflamed intestine, we first examined their effects on murine splenocytes. The addition of TGF- $\beta$ plus IL-6 drastically enhanced IL-17 production from the splenocytes (Fig. 1A). Of the five bacteria strains tested, $B$. infantis suppressed IL-17 production most potently. In parallel, TGF- $\beta$ plus IL-6 decreased the production of IL-27, which has been reported to suppress the generation of IL-17producing T cells, and $B$. infantis recovered IL-27 production (Fig. 1B). In contrast to B. infantis, the other bifidobacteria, $B$. bifidum and $B$. catenulatum, and the two tested lactobacilli did not exert a suppressive effect on IL-17 production in this study, although B. catenulatum increased IL-27 production. As shown in Fig. $1 \mathrm{C}$, the concentration of IL-17 was negatively correlated with that of IL-27 $(r=0.82)$ in TGF- $\beta$ plus IL-6-stimulated cultures. Thus, the IL-17/IL-27 axis was judged to be a reliable marker of Th17 status in the splenocytes.

IL-10 is important for the suppression of Th17 function in the splenocytes. To examine the mechanisms by which $B$. infantis suppressed IL-17 production in murine splenocytes, the effect of B. infantis on the cytokine profile [IL-2, IL-4, IL-5, IL-10 and IL-12 (p70)] of the splenocytes was evaluated using a cytokine array system, the Bio-Plex apparatus. As shown in Table I, cytokine production of Th1 (IL-12) and Th2 (IL-4, IL-5) was greatly suppressed by TGF- 3 plus IL-6. $B$. infantis recovered IL-5 and IL-12 production to some extent. Although the IL-10 concentration was not altered by the addition of TGF- $\beta$ plus IL- $6, B$. infantis significantly 
(A)

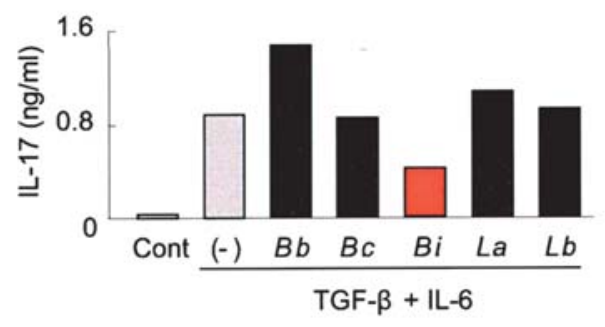

(B)

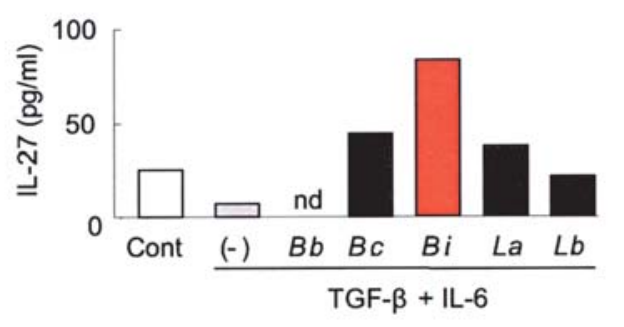

(C)

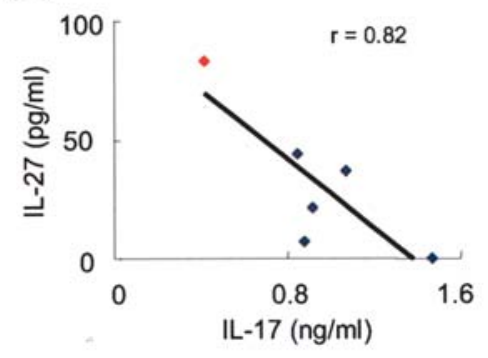

Figure 1. Effects of bifidobacteria and lactobacilli on IL-17 and IL-27 production in murine splenocytes. Spleen were removed from three mice, and the pooled splenocytes $\left(10^{7}\right.$ cells) were incubated with TGF- $\beta$ plus IL-6. B. bifidum (Bb), B. catenulatum (Bc), B. infantis (Bi), L. acidophilus (La), or L. bulgaricus ( $\mathrm{Lb})\left(10^{7}\right.$ cells each) were added to the culture. The culture alone (Cont) and the culture without the addition of bacteria (-) were included as controls. Culture supernatants $(n=3)$ were harvested, pooled and assayed for IL-17 (A) and IL-27 (B) concentrations. The relationship between IL-17 and IL-27 concentrations is shown in (C).

elevated the IL-10 concentration. Therefore, it was assumed that the induction of immunoregulatory IL-10 by $B$. infantis was, at least in part, important for suppressing the Th17 function in the splenocytes.

Effects of bifidobacteria on IL-17 and eotaxin production in DSS-induced intestinal inflammation. Next, we examined whether bifidobacteria suppressed IL-17 production in the ex vivo colon organ culture. We first stimulated the intestinal tract by the addition of TGF- $\beta$ plus IL- 6 as in the experiment using splenocytes. However, TGF- $\beta$ plus IL- 6 failed to enhance IL-17 production from the colon culture (Fig. 2A). Thus, to generate IL-17-producing cells, we used DSS, which is frequently used for the induction of acute colitis in vivo. As shown in Fig. 2A, DSS successfully induced IL-17 production from the culture in a dose-dependent manner. Because the IL-17 concentration in the $2 \%$ DSS-containing medium of the colon culture almost tripled, this DSS concentration was used for further analyses. In the colon culture, all bifidobacteria tested effectively suppressed IL-17 production.
(A)

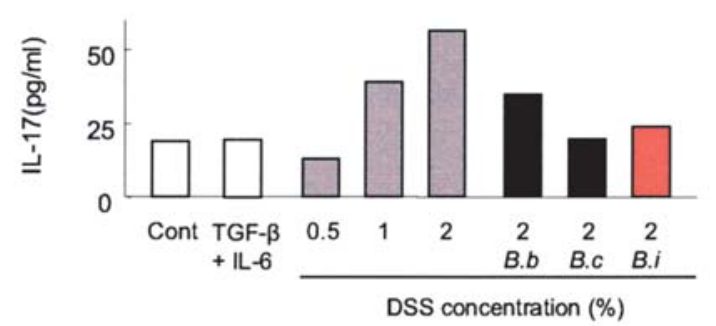

(B)

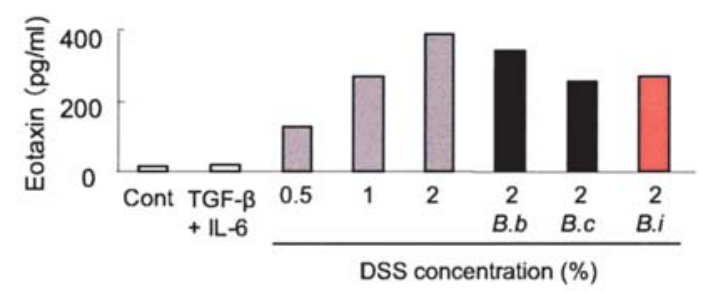

(C)

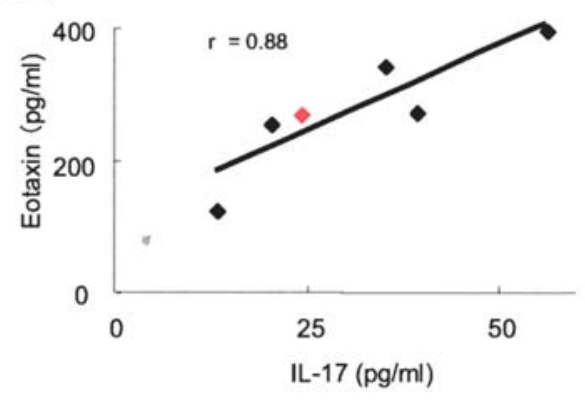

Figure 2. Effects of bifidobacteria on IL-17 and chemokine production in colon organ culture. Segments of the colon were removed from three mice, cut into strips and pooled. The strips were placed in a culture plate and incubated with TGF- $\beta$ plus IL- 6 or the indicated concentrations of DSS. B. bifidum (B.b.), B. catenulatum (B.c.), or B. infantis (B.i.) $\left(10^{5}\right.$ cells) were added to the culture under stimulation with $2 \%$ DSS. The culture alone (Cont) was included as a control. Culture supernatants of four strips were harvested and assayed for IL-17 (A) and eotaxin (B) concentrations. The relationship between IL-17 and the eotaxin concentrations is shown in (C). The results are representative of three independent experiments.

In contrast to the splenocytes, IL-27 production from the colon culture was not greatly altered even by the addition of $2 \%$ DSS; IL-27 concentrations of non-stimulated and $2 \%$ DSS-containing culture media were 7.2 and $5.5 \mathrm{pg} / \mathrm{ml}$, respectively.

In addition, we evaluated the level of eotaxin, a chemokine and an effector for tissue inflammation. As a result, little eotaxin production was observed in non-inflamed and in TGF- $\beta$ plus IL-6-stimulated intestines; however, DSS enhanced its production in the culture in a dose-dependent manner (Fig. 2B). All bifidobacteria tested effectively suppressed eotaxin production. As shown in Fig. 2C, the concentration of IL-17 was well correlated with that of eotaxin $(r=0.88)$ in DSS-treated cultures. Therefore, it was confirmed that bifidobacteria ameliorated DSS-induced inflammation in the colon culture.

$B$. infantis induced regulatory $I L-10$ in the inflamed intestine. We next examined the cytokine profile of the inflamed 
Table I. Effects of B. infantis on cytokine profile released from splenocytes.

\begin{tabular}{lrlc}
\hline & \multirow{2}{*}{ Control } & \multicolumn{2}{c}{ TGF- $\alpha+$ IL-6 } \\
\cline { 3 - 4 } & & \multicolumn{1}{c}{$(-)$} & B. infantis \\
\hline IL-2 & $141.9 \pm 3.0^{\mathrm{a}}$ & $55.8 \pm 26.2^{\mathrm{b}}$ & $19.6 \pm 1.9^{\mathrm{b}}$ \\
IL-4 & $9.1 \pm 0.5^{\mathrm{a}}$ & $0.86 \pm 0.31^{\mathrm{b}}$ & $0.90 \pm 0.09^{\mathrm{b}}$ \\
IL-5 & $9.2 \pm 1.3^{\mathrm{a}}$ & $0.34 \pm 0.11^{\mathrm{b}}$ & $0.69 \pm 0.03^{\mathrm{b}}$ \\
IL-10 & $15.5 \pm 0.7^{\mathrm{a}}$ & $15.7 \pm 2.5^{\mathrm{a}}$ & $31.3 \pm 1.3^{\mathrm{b}}$ \\
IL-12 (p70) & $3.7 \pm 0.1^{\mathrm{a}}$ & $0.36 \pm 0.04^{\mathrm{b}}$ & $1.1 \pm 0.1^{\mathrm{c}}$ \\
\hline
\end{tabular}

Data represent the mean $\pm \mathrm{SE}(\mathrm{pg} / \mathrm{ml})$ of three cultures. ${ }^{\mathrm{a}, \mathrm{b}, \mathrm{c}}$ Different letters indicate significantly difference between two groups $(\mathrm{p}<0.05)$.

intestine. For this experiment, we chose B. infantis, because it suppressed IL-17 production both in the splenocytes and in the colon culture effectively. We observed that the function of the regulatory cells was hampered by DSS, since the IL-10 concentration was greatly decreased by the addition of DSS (Table II). However, more noteworthy was that B. infantis elevated the IL-10 concentration to the non-stimulated level. Therefore, it was assumed that $B$. infantis suppressed IL-17 production in the inflamed intestine in response to IL-10 as observed in the splenocytes (Table I). Unfortunately, the concentration of IL-2, which is also a regulatory cytokine, was below the detection limit. The cytokine production of Th1 (IL-12) and Th2 (IL-4 and IL-5) was also suppressed by DSS, although the concentrations of IL-4 and IL-12 were very low. Nevertheless, it was assumed that $B$. infantis did not affect these cytokines in the colon culture.

\section{Discussion}

In this study, we clearly showed for the first time that $B$. infantis suppressed IL-17 production both in splenocytes and in colon organ culture. There were two reasons for choosing commensal bifidobacteria and probiotic lactobacilli for this study. First, these bacteria have been reported to have anti-inflammatory activities in certain inflammatory conditions $(17,18,21)$, although the relationships between the bacteria and IL-17 suppression have not been evaluated and discussed. Second, it is highly likely that these bacteria maintain homeostasis in our intestine as they actually live there. We used heat-killed bacteria rather than live bacteria in our experiments because we found that the vigorous growth of live bacteria in the splenocytes and colon organ culture would make proper evaluations difficult to carry out.

We clarified that one of the preventive effects of bifidobacteria on intestinal inflammation, which were clinically observed, can be explained by the inhibition of IL-17 production. Of the five tested bacteria strains, B. infantis exerted the most potent inhibitory activity on IL-17 production. A major role for IL-17 has now been described in various models of immune-mediated tissue injury, including organ-specific autoimmunity in the brain, heart, synovium, and intestines, allergic disorders of the lung and skin, and microbial infections of the intestines and the nervous system.
Table II. Effects of B. infantis on cytokine profile released from colon organ culture.

\begin{tabular}{lccc}
\hline & & \multicolumn{2}{c}{$2 \%$ DSS } \\
\cline { 3 - 4 } & Control & $(-)$ & B infantis \\
\hline IL-2 & nd & nd & nd \\
IL-4 & $0.45 \pm 0.08$ & nd & nd \\
IL-5 & $14.1 \pm 5.8^{\mathrm{a}}$ & $9.8 \pm 2.6^{\mathrm{a}}$ & $7.0 \pm 1.0^{\mathrm{a}}$ \\
IL-10 & $1.2 \pm 0.0^{\mathrm{a}}$ & $0.45 \pm 0.12^{\mathrm{a}}$ & $1.0 \pm 0.4^{\mathrm{a}}$ \\
IL-12 (p70) & $0.29 \pm 0.12^{\mathrm{a}}$ & $0.17 \pm 0.03^{\mathrm{a}}$ & $0.08 \pm 0.04^{\mathrm{a}}$ \\
\hline IL-17 & $8.7 \pm 1.4^{\mathrm{a}}$ & $19.3 \pm 2.1^{\mathrm{b}}$ & $10.9 \pm 0.8^{\mathrm{a}}$ \\
\hline
\end{tabular}

Data represent the mean $\pm \mathrm{SE}(\mathrm{pg} / \mathrm{ml})$ of three cultures. nd, not detected. ${ }^{a, b}$ Different letters indicate significantly difference between two groups $(\mathrm{p}<0.05)$.

A pathway named Th17 is now credited for causing and sustaining tissue damage in these diverse situations (7). Th17 cells are generated from naïve $\mathrm{T}$ cells, and Th17 cell differentiation is driven by stimuli such as TGF- $\beta$ plus IL- 6 (26). Indeed, TGF- $\beta$ plus IL-6 remarkably induced IL-17 production from murine splenocytes in this study (Fig. 1). However, the same concentrations of TGF- $\beta$ plus IL- 6 failed to stimulate IL-17 production from the colon culture; instead, DSS induced IL-17 production in a dose-dependent manner (Fig. 2). In response to DSS, dendritic cells might help naïve $\mathrm{T}$ cells to differentiate into Th17 cells in the colon culture (27).

In DSS-induced colitis, IL-6 plays a critical role, since the development of colitis is reduced in IL-6-deficient mice treated with DSS (28). IL-6 is one of the main cytokines secreted by lamina propria cells, and overproduction of IL-6 has been found in many types of colitis. Thus, it is assumed that the mechanism by which DSS induces IL-17 production might also be involved with IL-6. In this study, $B$. infantis was anticipated to inhibit the action of IL-6 in the colon culture as well as in the splenocytes. If $B$. infantis could inhibit IL-6 in the splenocytes, the Treg/Th17 balance would be shifted toward Treg as a result of added TGF- $\beta$. Further analyses are needed to clarify the IL-6-suppressing effect of $B$. infantis.

In the splenocytes, $B$. infantis induced IL-27 production while suppressing IL-17 production. Awasthi et al (15) showed that IL-27 potently enhanced IL-10 production in vitro. Indeed, in our study, $B$. infantis elevated the IL-10 concentration both in TGF- $\beta$ plus IL-6-stimulated splenocytes and in DSS-treated colon culture. Therefore, we concluded that $B$. infantis suppressed IL-17 production by inducing immunoregulatory IL-10 and IL-27 production. The anti-inflammatory effects of these types of bacteria have been explained by down-regulation of proinflammatory (e.g., IL-12 and TNF- $\alpha$ ) cytokine production and/or stimulation of antiinflammatory (e.g., IL-10) cytokine production (19). However, to the best of our knowledge, this is the first finding that $B$. infantis enhanced IL-27 production which subsequently led to the induction of IL-10 and the suppression of IL-17 production. To date, studies on the immunomodulatory effects of lactic acid bacteria have focused mainly on the effects on Treg and the Th1/Th2 balance. For example, some probiotic 
bacteria have been reported to suppress perennial allergic rhinitis (29), as one of the mechanisms involved in occurring pollinosis is the imbalance of Th1/Th2 (30,31). We anticipate that the Th17-inhibitory activity of probiotic and/or commensal bacteria will be further examined.

It should be noted that even 'heat-killed' bacteria suppressed IL-17 production in our study. The precise mechanism and the bacterial component(s) responsible for this activity remain unclear. It is generally accepted that bacterial components are recognized by members of the pattern recognition receptor (PRR) family such as TLRs (23) and Nod-like proteins $(32,33)$. In fact, some studies have shown that the TLR2 ligand PCSK ameliorated TNF- $\alpha$-induced intestinal barrier impairment in the human epithelial Caco- 2 cells $(20,24)$. It would be beneficial to investigate whether the recognition of bacterial components by the PRR family are involved in the inhibition of IL-17 production by bifidobacteria. It is also probable that bifidobacteria induce the apoptosis of Th17 cells or other IL-17-producing cells.

In conclusion, we established an ex vivo screening system of IL-17 regulation in the intestinal tract and showed for the first time that certain bifidobacteria suppress IL-17 production in response to recovering Treg activity. Whether IL-17 suppression is involved in the in vivo preventive effects of bifidobacteria in DSS-induced colitis remains to be examined. The present results further imply that these bacteria would be useful in the treatment of Th17-mediated diseases, although further evaluation is needed to confirm the effects of bifidobacteria on human cells or organs. We are also planning to screen bacteria from a wide bacterial library to identify those with more potent IL-17-inhibitory activity.

\section{Acknowledgements}

We thank Dr Seiji Kawamoto (Hiroshima University) for his valuable suggestions and Ms. Chisa Takahata for her technical assistance.

\section{References}

1. Xavier RJ and Podolsky DK: Unravelling the pathogenesis of inflammatory bowel disease. Nature 448: 427-434, 2007.

2. Carpenter HA and Talley NJ: The importance of clinicopathological correlation in the diagnosis of inflammatory conditions of the colon: histological patterns with clinical implications. Am J Gastroenterol 95: 878-896, 2000.

3. Liu Z, Jiu J, Liu S, Fa X, Li F and Du Y: Blockage of tumor necrosis factor prevents intestinal mucosal inflammation through down-regulation of interleukin-23 secretion. J Autoimmun 29: 187-194, 2007.

4. Sartor RB: Microbial influences in inflammatory bowel diseases. Gastroenterology 134: 577-594, 2008.

5. Kolls JK and Linden A: Interleukin-17 family members and inflammation. Immunity 21: 467-476, 2004.

6. Bettelli E, Oukka $M$ and Kuchroo VK: $\mathrm{T}_{\mathrm{H}^{-1}} 17$ cells in the circle of immunity and autoimmunity. Nat Immunol 8: 345-350, 2007.

7. Steinman L: A brief history of $T_{H} 17$, the first major revision in the $T_{H} 1 / T_{H} 2$ hypothesis of $T$ cell-mediated tissue damage. Nat Med 13: 139-145, 2007.

8. Ivanov II, McKenzie BS, Zhou L, et al: The orphan nuclear receptor RORgammat directs the differentiation program of proinflammatory IL-17+ T helper cells. Cell 126: 1121-1133, 2006.

9. Fossiez F, Djossou O, Chomarat $\mathrm{P}$, et al: $\mathrm{T}$ cell interleukin-17 induces stromal cells to produce proinflammatory and hematopoietic cytokines. J Exp Med 183: 2593-2603, 1996.
10. Laurence A, Tato CM, Davidson TS, et al: Interleukin-2 signaling via STAT5 constrains T helper 17 cell generation. Immunity 26: 371-381, 2007.

11. Kleinschek MA, Owyang AM, Joyce-Shaikh B, et al: IL-25 regulates Th17 function in autoimmune inflammation. J Exp Med 204: 161-170, 2007.

12. Batten M, Li J, Yi S, et al: Interleukin 27 limits autoimmune encephalomyelitis by suppressing the development of interleukin 17-producing T cells. Nat Immunol 7: 929-936, 2006.

13. Stumhofer JS, Silver JS, Laurence A, et al: Interleukins 27 and 6 induce STAT3-mediated T cell production of interleukin 10. Nat Immunol 8: 1363-1371, 2007.

14. Fitzgerald DC, Zhang GX, El-Behi M, et al: Suppression of autoimmune inflammation of the central nervous system by interleukin 10 secreted by interleukin 27 -stimulated T cells. Nat Immunol 8: 1372-1379, 2007.

15. Awasthi A, Carrier Y, Peron JP, et al: A dominant function for interleukin 27 in generating interleukin 10-producing antiinflammatory T cells. Nat Immunol 8: 1380-1389, 2007.

16. McGeachy MJ, Bak-Jensen, KS, Chen Y, Tato CM, Blumenschein W, McClanahan T and Cua DJ: TGF-beta and IL-6 drive the production of IL-17 and IL-10 by T cells and restrain T(H)-17 cell-mediated pathology. Nat Immunol 8: 1390-1397, 2007.

17. Rioux KP and Fedorak RN: Probiotics in the treatment of inflammatory bowel disease. J Clin Gastroenterol 40: 260-263, 2006.

18. Lorea Baroja M, Kirjavainen PV, Hekmat S and Reid G: Antiinflammatory effects of probiotic yogurt in inflammatory bowel disease patients. Clin Exp Immunol 149: 470-479, 2007.

19. Di Giacinto C, Marinaro M, Sanchez M, Strober W and Boirivant M: Probiotics ameliorate recurrent Th1-mediated murine colitis by inducing IL-10 and IL-10-dependent TGFbeta-bearing regulatory cells. J Immunol 174: 3237-3246, 2005.

20. Miyauchi E, Morita H, Okuda J, et al: Cell wall fraction of Enterococcus hirae ameliorates TNF- $\alpha$-induced barrier impairment in the human epithelial tight junction. Lett Appl Microbiol 46: 469-476, 2008.

21. Resta-Lenert S and Barrett KE: Probiotics and commensals reverse TNF-alpha- and IFN-gamma-induced dysfunction in human intestinal epithelial cells. Gastroenterology 130: 731-746, 2006.

22. Ko JS, Yang HR, Chang JY and Seo JK: Lactobacillus plantarum inhibits epithelial barrier dysfunction and interleukin-8 secretion induced by tumor necrosis factor- $\alpha$. World J Gastroenterol 13: 1962-1965, 2007.

23. Trinchieri G and Sher A: Cooperation of Toll-like receptor signals in innate immune defence. Nat Rev Immunol 7: 179-190, 2007.

24. Cario E, Gerken G and Podolsky DK: Toll-like receptor 2 controls mucosal inflammation by regulating epithelial barrier function. Gastroenterology 132: 1359-1374, 2007.

25. Lee J, Mo JH, Katakura K, et al: Maintenance of colonic homeostasis by distinctive apical TLR9 signalling in intestinal epithelial cells. Nat Cell Biol 8: 1327-1336, 2006.

26. Kimura A, Naka T and Kishimoto T: IL-6-dependent and -independent pathways in the development of interleukin 17producing T helper cells. Proc Natl Acad Sci USA 104: 12099-12104, 2007.

27. Denning TL, Wang YC, Patel SR, Williams IR and Pulendran B: Lamina propria macrophages and dendritic cells differentially induce regulatory and interleukin 17-producing $\mathrm{T}$ cell responses. Nat Immunol 8: 1086-1094, 2007.

28. Suzuki A, Hanada T, Mitsuyama K, et al: CIS3/SOCS3/SSI3 plays a negative regulatory role in STAT3 activation and intestinal inflammation. J Exp Med 193: 471-481, 2001.

29. Ishida Y, Nakamura F, Kanzato H, et al: Clinical effects of Lactobacillus acidophilus strain L-92 on perennial allergic rhinitis: a double-blind, placebo-controlled study. J Dairy Sci 88: 527-533, 2005.

30. Tanabe S, Shibata R and Nishimura T: Hypoallergenic and T cell reactive analogue peptides of bovine serum albumin, the major beef allergen. Mol Immunol 41: 885-890, 2004.

31. Tanabe S: Epitope peptides and immunotherapy. Curr Protein Pept Sci 8: 109-118, 2007.

32. Strober W, Murray PJ, Kitani A and Watanabe T: Signalling pathways and molecular interactions of NOD1 and NOD2. Nat Rev Immunol 6: 9-20, 2006.

33. Fritz JH, Ferrero RL, Philpott DJ and Girardin SE: Nod-like proteins in immunity, inflammation and disease. Nat Immunol 7: $1250-1257,2006$ 